\title{
Design and Evaluation of Autonomous Hybrid Frequency-Voltage Sensitive Load
} Controller

Douglass, Philip James; Garcia-Valle, Rodrigo; Sossan, Fabrizio; Østergaard, Jacob; Nyeng, Preben

Published in:

Proceedings of Innovative Smart Grid Technologies (ISGT) Europe 2013

Publication date:

2013

Link back to DTU Orbit

Citation $(A P A)$ :

Douglass, P. J., Garcia-Valle, R., Sossan, F., Østergaard, J., \& Nyeng, P. (2013). Design and Evaluation of Autonomous Hybrid Frequency-Voltage Sensitive Load Controller. In Proceedings of Innovative Smart Grid Technologies (ISGT) Europe 2013 IEEE.

\section{General rights}

Copyright and moral rights for the publications made accessible in the public portal are retained by the authors and/or other copyright owners and it is a condition of accessing publications that users recognise and abide by the legal requirements associated with these rights.

- Users may download and print one copy of any publication from the public portal for the purpose of private study or research.

- You may not further distribute the material or use it for any profit-making activity or commercial gain

- You may freely distribute the URL identifying the publication in the public portal 


\section{Design and Evaluation of Autonomous Hybrid Frequency-Voltage Sensitive Load Controller}

\author{
Philip J. Douglass, Rodrigo Garcia-Valle, Fabrizio Sossan, Jacob Østergaard \\ Technical University of Denmark, \\ Elektrovej-Building 325, \\ 2800 Kgs. Lyngby, Denmark \\ Email: \{pjdo, rgv, faso, joe\}@elektro.dtu.dk
}

\author{
Preben Nyeng \\ Energinet.dk, \\ Tonne Kjærsvej 65, \\ 7000 Fredericia, Denmark \\ Email: pny@energinet.dk
}

\begin{abstract}
The paper introduces an algorithm for control of autonomous loads without digital communication interfaces to provide both frequency regulation and voltage regulation services. This hybrid controller can be used to enhance frequency sensitive loads to mitigate line overload arising from reduced load diversity. Numerical simulations of the hybrid controller in a representative distribution system show the peak system load was reduced by $12 \%$ compared to a purely frequency sensitive load controller.
\end{abstract}

\section{INTRODUCTION}

Time-sensitive and geographically distributed control of today's power system is achieved by the use of local control loops that measure system parameters and act upon them autonomously. Examples include speed droop governors, voltage regulators in synchronous generators, and on-load tap changing transformers.

Generators have primary responsibility for maintaining system frequency and voltage within specified limits with $P-f$ and $Q-V$ droop control. In large power systems with inductive transmission lines, these two control objectives are decoupled, but in the general case (including micro-grids with resistive lines) the two objectives are interrelated [1].

Local control loops are also being applied to distributed generation (DG) to allow small generation units to coordinate their actions and contribute the stabilizing system frequency and voltage without the overhead of a reliable data communications network. For example, photo-voltaic (PV) inverters connected to low voltage distribution systems in Germany are required to implement $P-f$ droop control to curtail active power when system frequency rises above $50.2 \mathrm{~Hz}$ [2], and research indicates that curtailing active power in response to voltage rises can increase feasible DG penetration [3].

Using loads to regulate system parameters through Under-Frequency Load Shedding (UFLS) and UnderVoltage Load Shedding (UVLS) is well established, but only as a last resort defense. Loads with inherent flexibility, such as thermostat controlled loads (TCLs), can be designed so power consumption is shifted in time without compromising the quality of energy service provided. These loads have the potential to modulate their active power consumption and contribute to stabilizing system frequency and voltage as a part of normal operation [4]. Continuing advances in micro-electronics allow sufficiently accurate measurement of system frequency and voltage by the low-cost microcontrollers typically found in white goods appliances. These measurements can provide input to load controllers that allow loads to participate in frequency and voltage regulation autonomously without reliance on real-time digital communications. Autonomous load controllers can be deployed in a "fit and forget" fashion or they may be built with digital communications interfaces to allow remote changes to configuration parameter values.

While autonomous frequency sensitive loads (FSL) have matured to be candidates for mass-deployment [5], the local consequences of reduced FSL load diversity that unavoidably results from providing frequency regulation service has not been addressed. Specifically, synchronizing loads in response to frequency variations threatens to cause line overload in congested distribution systems. To the extent that these constraints are reflected in RMS voltage deviations, autonomous loads can use RMS voltage as an input to a hybrid controller that dampens the frequency response if and only if line overload occurs.

This paper describes a novel autonomous load control algorithm that contributes to stabilizing system frequency and RMS voltage. The performance of this controller is analyzed by simulating its behavior when controlling TCLs in a representative distribution system.

This paper is organized as follows. Section II describes autonomous load control algorithms including the proposed hybrid frequency-voltage sensitive load controller. Section III describes the setup of the simulation environment and Section IV presents the results of the simulations. Finally, section V concludes the paper.

\section{Autonomous LoAd CONTROL}

\section{A. General Autonomous TCL Model}

The autonomous load controller, which is a generalized version of the frequency sensitive load controller in [7], operates in the system shown in Fig. 1. The load controller samples the energy-carrying voltage waveform $v$, and the 


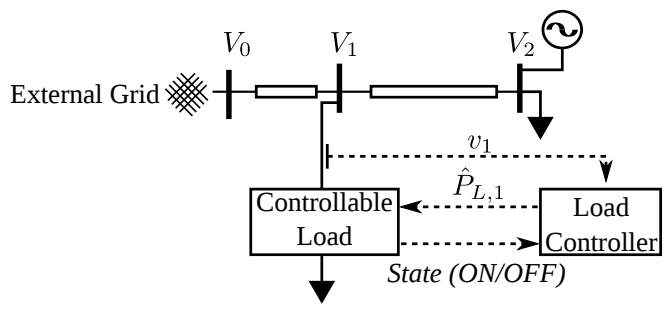

Figure 1. Oneline system diagram showing controllable load and DG in a radial feeder [6]. Dashed lines represent control signal paths.

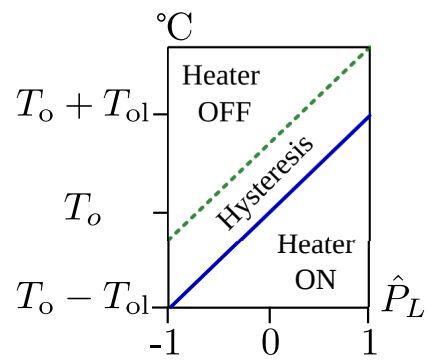

Figure 2. Dependance of thermostat setpoint on $\hat{P}_{L}$ in heating application [6]. Y-axis is process temperature i.e. of water in hot water tank. The heater turns ON when the temperature falls below the solid line, and turns OFF when the temperature rises above the dashed line.

state of the load (ON/OFF). The controller output $\hat{P}_{\mathrm{L}}$ is the desired load power consumption, normalized to lie between $[-1,1]$ where -1 represents no power consumption, and 1 represents full power. The desired power consumption $\hat{P}_{\mathrm{L}}$ is given to a controllable load that will attempt to comply with the request, within the constraints imposed by the final energy conversion process.

This paper will examine the use of TCLs for demand response because they represent a large, and potentially controllable, load in residential areas. The thermostat setpoint $T_{\mathrm{s}}$ is the result of linearly mapping $\hat{P}_{\mathrm{L}}$ to an offset to the user-given thermostat temperature setpoint $T_{\mathrm{o}}$, up (down) to the offset limit $T_{\mathrm{ol}}$ :

$$
T_{\mathrm{s}}=T_{\mathrm{o}}+T_{\mathrm{ol}} \hat{P}_{\mathrm{L}}
$$

The thermostat state as a function of process temperature and thermostat offset is shown in Fig. 2.

\section{B. Frequency Sensitive Loads}

Claims that frequency sensitive loads (FSLs) are a large, feasible and low cost resource for primary frequency regulation are supported by analysis [7] and experiments [8].

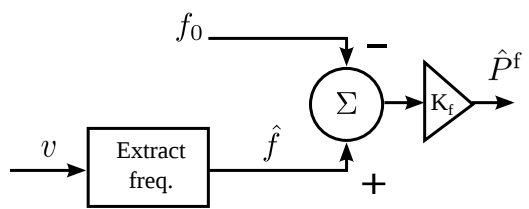

Figure 3. Frequency Response subsystem.
This has motivated recent efforts by European transmission system operators to consider mandating integration of frequency response into TCLs [5].

Fig. 3 shows a block diagram of the FSL controller that produces an output $\hat{P}^{\mathrm{f}}$ proportional to the measured AC frequency $\hat{f}$, where the sensitivity is given by the gain constant $\mathrm{K}_{\mathrm{f}}$. The reader is referred to existing literature [9], [10] for a full discussion of the properties of FSLs, the salient issue for this work is the reduction in FSL diversity caused by the frequency response. The present practice of distribution system planners is to assume a random distribution of the internal state of loads and apply a coincidence factor to de-rate the installed capacity of a load class to the maximum expected aggregate load. Field tests of FSL for space heating show that in certain weather conditions the aggregate load approaches installed capacity [8] during high frequency events.

In [11] the authors propose a protocol for quickly restoring FSL diversity after a responding to an event, but the protocol does not alleviate the local transmission bottle-necks the arise during the event response itself. A blanket reduction of the capacity of FSLs is suboptimal because congested conditions may happen only in a few locations for a short time.

\section{Voltage Sensitive Loads}

The purpose of the voltage sensitive loads (VSL) controller is to regulate system voltage by modulating the power consumption of flexible loads. In networks where system load and RMS voltage are inversely correlated, a VSL that reduces power consumption when voltage is low is acting to increase total load diversity. Fig. 4 shows a block diagram of the voltage-sensitive load (VSL) controller, full details of this algorithm can be found in [6], a brief description is given here.

The controller is given a RMS voltage measurement and calculates a short-term moving average $\hat{v}$ over a time frame of seconds (the exact value is a configurable parameter), a function that filters out measurement noise and transient faults. This short-term average is then subtracted from the long-term average voltage value $\bar{v}$ giving the relative voltage difference $\Delta V$. This difference is then scaled by a gain factor $\mathrm{G}$ to determine the desired power consumption of the load $\hat{P}^{\mathrm{V}}$.

The long-term average voltage value $\bar{v}$ is found by again using a moving average over a time period of hours to days (exact value is configurable, but it must be much greater than short-term value). Two long-term average voltage values are found: one long-term average of voltage measurements taken when the device is $\mathrm{ON}$, and one when the device is OFF. Switching between the two values based on the state of the device compensates for the changes in voltage caused by the device itself.

The controller "auto-tunes" G, thus normalizing the voltage response relative to magnitude of observed voltage variations. The long-term moving variance of $\Delta V$ is found 


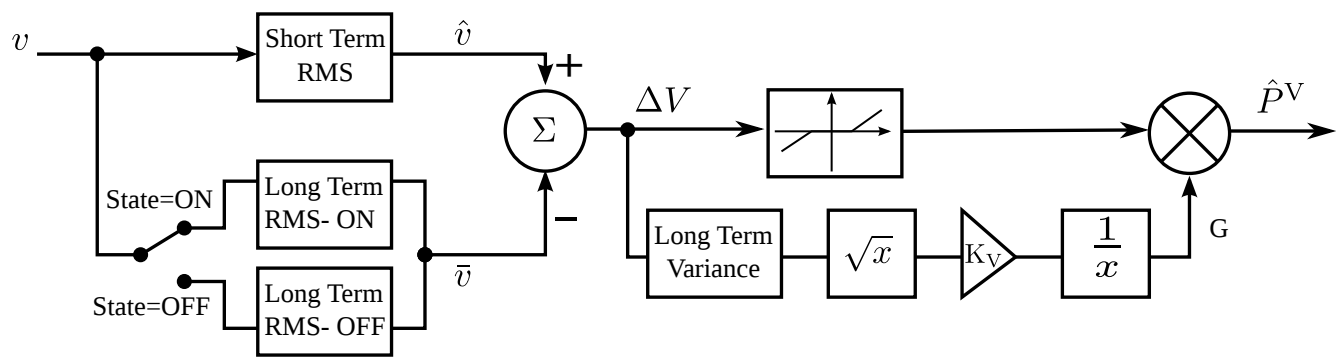

Figure 4. Voltage Response subsystem [6].

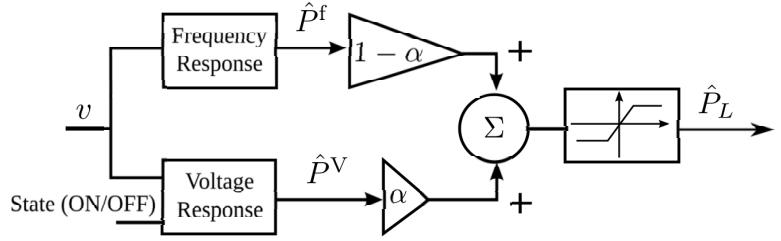

Figure 5. Block diagram of hybrid frequency and voltage sensitive load controller. Weighting factor $\alpha$ is limited to be between $[0,1]$.

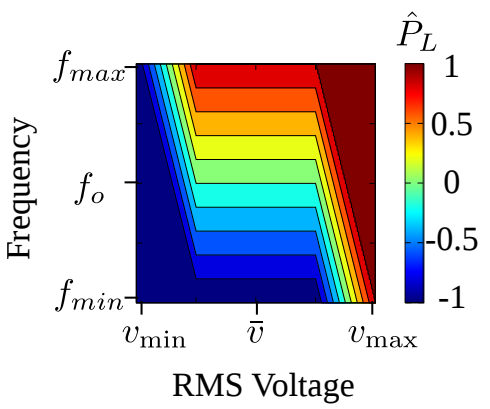

Figure 6. Dependance of $\hat{P}_{L}$ on voltage and frequency. The voltage response has a deadband around the expected value $\bar{v}$, while the frequency response has a continuously linear response.

over a time span equal to that used for calculating $\bar{v}$. The standard deviation $\sigma$ is found as the square root of the variance, then multiplied by a fixed value $\mathrm{K}_{\mathrm{V}}$ and inverted to give $\mathrm{G}$.

One extension is made to the algorithm introduced in [6], the addition of a deadband which holds the controller output at zero for values of $\Delta V$ below a given threshold.

\section{Hybrid Frequency-Voltage Sensitive Loads}

The load control algorithm introduced in this work is shown with a high-level block diagram in Fig. 5. The output is the weighted sum, with weighting factor $\alpha$, of a frequency response $\hat{P}^{f}$ and a voltage response $\hat{P}^{\mathrm{V}}$, each of which has been described earlier in this section:

$$
\hat{P}_{\mathrm{L}}=(1-\alpha) \hat{P}^{\mathrm{f}}+\alpha \hat{P}^{\mathrm{V}}
$$

The output $\hat{P}_{\mathrm{L}}$ is limited to be between $[-1,1]$. The optimal value of $\alpha$ will depend on the relative importance of frequency and voltage regulation in a specific power system.
The controller output value is shown graphically as a function of the outputs of two subsystems in Fig. 6, where the parameters of the voltage response are chosen such that there is a deadband around the long-term average voltage value $\bar{v}$.

\section{Simulation CONFIGURATiOn}

The performance of the proposed load controller algorithm is evaluated with numerical simulations using GridLAB-D [12] on a feeder representing a typical North American distribution system. GridLAB-D is a discrete event simulation platform that contains detailed models of electrical distribution system components and loads, together with weather data, and a framework for collecting statistics about the state of the network and loads. Unbalanced voltage values are found with high precision because the simulator calculates the full $3 \times 3$ mutual impedance matrix for each component as given in [13]. As an opensource project, it is amenable to modification, and the controller described in section II-D was implemented in $\mathrm{C}++$ and compiled into the software.

Simulations were run over 10 days, with a minimum time step of $10 \mathrm{~s}$ for three scenarios: purely FSL, Hybrid and a base case where TCL setpoints were held constant.

\section{A. Distribution Network Model}

The network model is taken from [14] where typical North American network topologies were created from a survey of operating networks. The network contains a mix overhead lines, underground cables, unbalanced laterals, 1175 residences, 750 transformers, and a total of 1900 busses. The uncontrolled conventional loads in the system are represented as HVAC loads with a heat load synthesized from typical weather conditions of the Pacific Northwest in January, and ZIP loads (constant impedance, constant current, and constant power) that follow a preset schedule derived from the daily demand patterns observed in the USA. House parameters such as size, indoor temperature preference, and insulation were subject to a uniform distribution, for full details see [15].

Distributed generation in the form of PV was added to each house in the distribution system. The size of the PV systems were chosen so they produced in aggregate approximately the same amount of energy as the water heaters consumed over the test period. PV production time 
series were derived from data taken in April from a $7 \mathrm{~kW}$ PV system in our lab in Denmark and scaled to the size of each residential system in the simulation, with spacial diversity created by randomly assigning each residence to 6 groups and skewing production profiles by $10 \mathrm{~s}$ between each group.

System frequency was generated from measurements taken in the Nordic power system as part of a field experiment [8]. Using a pre-recorded frequency time series simplified the simulation by preventing the changes in load from effecting frequency values.

\section{B. Thermostat Controlled Load Model}

In the simulations the controlled load is modeled as a hot water heater. Model parameters such as water heater power, capacity, thermostat setpoint, thermostat deadband and insulation were subject to a random distribution representing typical values found in the USA. The water demand of each household was constant at $q[t]=57 \mathrm{l} / \mathrm{hr}$ representing mean household water consumption, a constant demand assumes a decoupling of the energy demand to heat water and the time of water use. The single phase resistive heating element was modeled as a constant impedance load and the inlet water temperature was fixed to $T_{\mathrm{in}}[t]=15.5^{\circ} \mathrm{C}$. Water temperature in the tank was modeled by a first order discrete equation

$$
\begin{aligned}
T_{\mathrm{w}}[t+1]=\frac{1}{C}\left[\left(T_{\mathrm{o}}[t]-T_{\mathrm{w}}[t]\right) U_{\mathrm{a}}+\right. \\
\left.w[t] Q+q[t]\left(T_{\mathrm{in}}[t]-T_{\mathrm{w}}[t]\right)\right]
\end{aligned}
$$

where the water temperature $T_{\mathrm{w}}$ at time $t$ depends on the ambient temperature $T_{\mathrm{o}}$, the water temperature at the previous timestep, the thermal conductance of the tank jacket $U_{\mathrm{a}}$, the ON/OFF signal from the thermostat $w$, the gain of the heating element $Q$, the water demand $q$, and the heat capacitance of the full tank $C$. The temperature of the hot water was modeled as a single body, neglecting the thermocline that arises in real tanks.

\section{Controller Configuration}

Simulations were run for a base case with static thermostat settings $\left(T_{\mathrm{ol}}=0\right)$, scenarios with purely FSLs $(\alpha=0)$, and a balanced hybrid configuration $(\alpha=0.5)$. In the FSL and hybrid scenarios, the load controllers are configured with a maximum temperature offset of $T_{\mathrm{ol}}=3{ }^{\circ} \mathrm{C}$. The gain of the purely FSL controller was $K_{\mathrm{f}}=-30{ }^{\circ} \mathrm{C} / \mathrm{Hz}$. In the hybrid controller, the frequency gain was increased to $K_{\mathrm{f}}=-73{ }^{\circ} \mathrm{C} / \mathrm{Hz}$. The voltagesensitive controller had a short-term average smoothing constant of 1/60 and a long-term average smoothing constant of $1 / 43200$. The deadband was set to one standard deviation $\sigma$, and the voltage gain $\mathrm{K}_{\mathrm{V}}$ chosen so the controller saturated when $\Delta V=2.5 \sigma$.
Table I

Performance of Base Case, FSL and Hybrid Controller

\begin{tabular}{|l|r|r|r|}
\hline Parameter & Base & FSL & Hybrid \\
\hline Ave. W.H. Power & $757 \mathrm{~kW}$ & $781 \mathrm{~kW}$ & $781 \mathrm{~kW}$ \\
Ave. $\hat{P}_{\mathrm{L}}$ & n.a. & -0.001 & -0.008 \\
Ave. Daily Max. Load & $3925 \mathrm{~kW}$ & $7086 \mathrm{~kW}$ & $6251 \mathrm{~kW}$ \\
W.H. power at Max. & $838 \mathrm{~kW}$ & $4090 \mathrm{~kW}$ & $3409 \mathrm{~kW}$ \\
Mean Losses & $99 \mathrm{~kW}$ & $107 \mathrm{~kW}$ & $105 \mathrm{~kW}$ \\
Ave. Daily Min. V & 0.989 p.u. & 0.975 p.u. & 0.979 p.u. \\
\hline
\end{tabular}

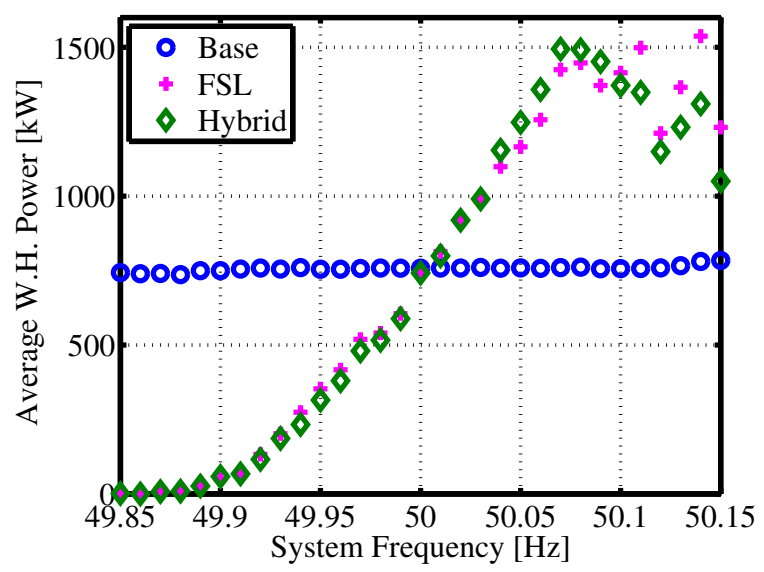

Figure 7. Average water heater power as a function of system frequency.

\section{Simulation Results}

First, the frequency response of the water heaters is evaluated by grouping each sample of aggregate power consumption by system frequency. The average water heater power as a function of frequency is shown in Fig. 7. The frequency response of the FSL controller matched closely the frequency response of the hybrid controller up until around $50.1 \mathrm{~Hz}$ when both controllers saturate.

Table I summarizes key performance statistics of the system. In both the FSL case and hybrid case, the power consumption of the TCLs increased by $3 \%$ compared to the base case, even though the thermostat offset had a slight negative bias. This is because the power consumption of the TCLs is asymmetrical with respect to thermostat offset.

The large amount of FSL greatly worsens the average of the daily peak power consumption measured at the external grid connection from under $4 \mathrm{MW}$ in the base case to over 7 MW. Substituting the FSL with the hybrid controller reduces the peak power to $6.25 \mathrm{MW}$, an improvement of $12 \%$ over the purely frequency sensitive controller, but still significantly worse than the base case. Looking at the power consumption of the water heaters at the daily peak load, the hybrid controller reduced the power of the water heaters at the peak load by $16 \%$ compared to the FSL. The average of daily minimum voltages is lowest with the FSL, improved with the hybrid controller, but best in the base case.

A typical time series showing the frequency response, 


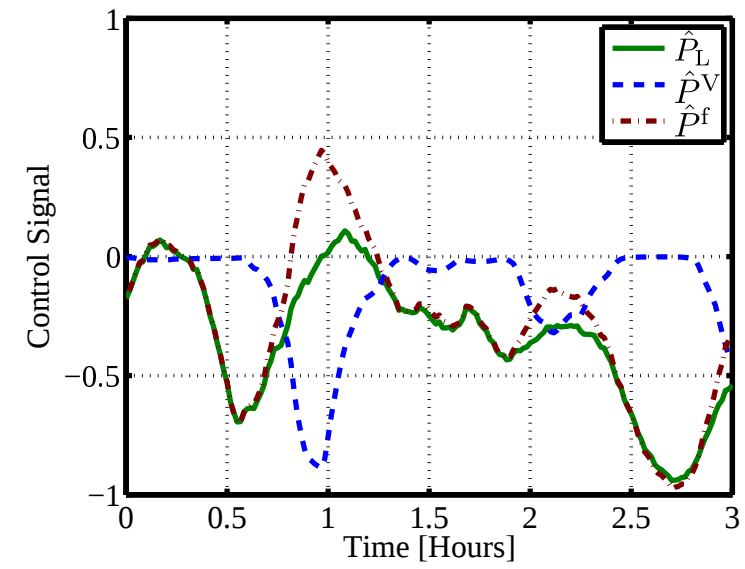

Figure 8. Representative time series of hybrid control $\hat{P}_{\mathrm{L}}$ signal and the voltage $\hat{P}^{\mathrm{V}}$ and frequency $\hat{P}^{\mathrm{f}}$ components.

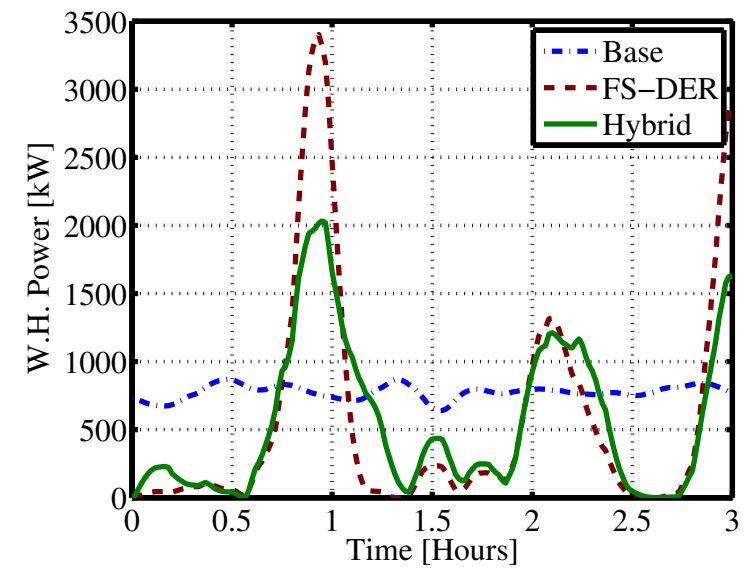

Figure 9. Time series of aggregate water heater power consumption for base case, FSL and hybrid controller.

voltage response, and the combined hybrid response is shown in Fig. 8. It shows in the first half-hour the voltage response lies in the deadband. Around the one hour mark the voltage response moves in the opposite direction as the frequency response, dampening the frequency response. In the beginning of the second hour, the voltage response has the same sign as the frequency response reenforcing the response. The aggregate power consumption of the water heaters shown for the base case, FSL and hybrid controller for the same time period in Fig. 9. The hybrid controller has a similar load shape as the FSL but the hybrid controller has clipped the sharp spike in demand at the end of the first hour. The base case shows very little variation in the aggregate water heater power consumption, so it is apparent that any frequency response would worsen the TCL load diversity.

\section{CONCLUSION}

An autonomous hybrid frequency-voltage sensitive load controller was introduced and analyzed in this paper. The hybrid controller is a linear combination of two subsystems: a frequency response that modulates load power in proportion to system frequency, and a voltage response that modulates load power in proportion in relative deviations in voltage. The hybrid controller allows frequency sensitive loads providing frequency regulation to be integrated into congested distribution systems by moderating the frequency response when voltage deviations indicate congestion in the distribution system.

Simulations in GridLAB-D show that the introduction of frequency sensitive loads (FSL) significantly increases the system's peak load compared to a base case. A hybrid controller with a comparable frequency response reduced the system's peak load by $12 \%$ compared to the FSLs. The minimum voltage levels observed in the feeder were similarly better for the hybrid controller relative to the FSL, though both voltage and peak power levels were not restored to the level of the base case.

\section{REFERENCES}

[1] K. De Brabandere, B. Bolsens, J. Van den Keybus, A. Woyte, J. Driesen, and R. Belmans, "A voltage and frequency droop control method for parallel inverters," Power Electronics, IEEE Transactions on, vol. 22, no. 4, pp. 1107-1115, 2007.

[2] VDE, "Application Guide VDE-AR-N 4105: Generators in the low voltage distribution network," 1 Aug. 2010. [Online]. Available: http://www.vde.com/de/fnn/arbeitsgebiete/seiten/n4105.aspx

[3] T. Sansawatt, L. Ochoa, and G. Harrison, "Integrating distributed generation using decentralised voltage regulation," in Power and Energy Society General Meeting, 2010 IEEE, july 2010, pp. 1-6.

[4] F. Marra, "Electric Vehicles Integration in the Electric Power System with High Wind Penetration - the Charge/Discharge infrastructure," Ph.D. dissertation, Technical University of Denmark, Mar. 2013.

[5] ENTSO-E, "Draft Demand Connection Codes," 27 Jun. 2012.

[6] P. J. Douglass, R. Garcia-Valle, O. C. Tudora, and J. Østergaard, "Mitigating Congestion in Distribution Networks with Voltage Sensitive Load Controllers," submitted to Smart Grid, IEEE Trans. on, 2013.

[7] Zhao Xu, J. Østergaard, and M. Togeby, "Demand as frequency controlled reserve," Power Systems, IEEE Transactions on, vol. 26, no. 3 , pp. $1062-1071$, aug. 2011.

[8] P. Douglass, R. Garcia-Valle, P. Nyeng, J. Østergaard, and M. Togeby, "Smart Demand for Frequency Regulation: Experimental Results," to appear in Smart Grid, IEEE Transactions on, 2013

[9] J. Short, D. Infield, and L. Freris, "Stabilization of grid frequency through dynamic demand control," Power Systems, IEEE Transactions on, vol. 22, no. 3, pp. $1284-1293$, aug. 2007.

[10] M. Donnelly, S. Mattix, D. Trudnowski, and J. Dagle, "Autonomous demand response for primary frequency regulation," Pacific Northwest National Laboratory, Tech. Rep., Jan. 2012.

[11] N. A. Sinitsyn, S. Kundu, and S. Backhaus, "Safe protocols for generating power pulses with heterogeneous populations of thermostatically controlled loads," Energy Conversion and Management, vol. 67, no. 0, pp. $297-308,2013$.

[12] D. Chassin, K. Schneider, and C. Gerkensmeyer, "Gridlab-d: An open-source power systems modeling and simulation environment,' in Transmission and distribution conference and exposition, 2008. ieee/pes, april 2008, pp. $1-5$.

[13] W. Kersting, Distribution System Modeling and Analysis, ser. The electric power engineering series. Taylor \& Francis, 2012.

[14] K. Schneider, Y. Chen, D. Engle, and D. Chassin, "A Taxonomy of North American Radial Distribution Feeders," in Power Energy Society General Meeting, 2009. PES '09. IEEE, 2009, pp. 1-6.

[15] "GridLAB-D Residential Module User's Guide," 2013. [Online]. Available: http://sourceforge.net/apps/mediawiki/gridlab-d/ 